\section{Production of Colchicine-induced Autotetraploids in Pummelo (Citrus grandis Osbeck) through Indirect Organogenesis}

\author{
Jude W. Grosser, Divya Kainth, and Manjul Dutt ${ }^{1}$ \\ University of Florida, Institute of Food and Agricultural Sciences, Citrus \\ Research and Education Center, 700 Experiment Station Road, Lake Alfred, \\ FL 33850
}

Additional index words. Citrus paradisi, flow cytometry, interploid crosses, seedlessness

\begin{abstract}
Colchicine-induced stable autotetraploid plants were recovered through indirect organogenesis from stem sections of in vitro-grown zygotic seedlings of pink/red-fleshed pummelo (Citrus grandis L. Osbeck) selections cybrid Hirado Buntan (C-HBP) (pink), 5-1-99-3 (dark pink), and HBJL-5 (red), all derived from Hirado Buntan pink pummelo. Multiple shoot induction was achieved through indirect organogenesis from the callus produced from the cut ends of the treated explants. Ploidy levels of regenerated plantlets were determined through flow cytometry at a stage when recovered shoots had one to two expanded leaves. Recovered tetraploids proved to be stable after 2 years in the field. As expected, higher colchicine concentrations and treatment durations decreased the survival rates of the regenerated plantlets. Colchicine concentrations of $0.05 \%$ and $0.1 \%$ produced the most tetraploids; of the 19 total tetraploids produced, 10 were produced from the treatment with a colchicine concentration of $0.1 \%$ and six were produced from treatment with a concentration of $0.05 \%$. After flowering, these stable pink/red-fleshed tetraploid plants generated should be useful as breeding parents in our grapefruit/pummelo improvement program, especially if any show canker tolerance or reduced furanocoumarins. Use of monoembryonic tetraploids in interploid citrus crosses eliminates the need for embryo rescue to recover seedless triploid progeny; this research expands our pool of available high-quality monoembryonic tetraploid parents.
\end{abstract}

Grapefruit (Citrus paradisi Macf.) is an important crop in Florida and is grown for fresh fruit and juice. Florida contributes almost $30 \%$ of the world grapefruit production. Grapefruit is believed to have originated in Barbados as an accidental cross between a pummelo ( $C$. grandis Osbeck) and a sweet orange [Citrus sinensis (L.) Osbeck] (Barrett and Rhodes, 1976; Gmitter, 1995; Scora, 1975; Scora et al., 1982). The increasing competition and popularity of grapefruit in international markets have stimulated grapefruit breeders to develop new varieties that can meet consumers' demands.

Grapefruit vary in flesh color with white, pink, and red cultivars being available. The pink- and red-fleshed varieties are most popular and earn higher prices in the markets. Another highly desired trait in commercial grapefruit cultivars these days is seedlessness. Seedless varieties of fruits like banana, watermelon, grapes, and plantain are available in the market and sell more than their seedy counterparts. Seedlessness has gained importance in citrus in the recent past. Seediness is causing problems in the acceptance of the fruit in the local or international markets

Received for publication 17 Sept. 2013. Accepted for publication 6 May 2014.

${ }^{1}$ To whom reprint request should be addressed; e-mailmanjul@ufl.edu.
Soost and Cameron, 1975). However, when the tetraploid female parent is a polyembryonic cultivar, the hybrid embryo needs to be rescued under sterile conditions and has to be grown in vitro. This is performed to avoid the suppression of the zygotic hybrid triploid embryo by the dominating nucellar embryos that are present. This technique is not cost- or labor-effective and lowers the effectiveness of triploid breeding programs. On the other hand, use of a tetraploid monoembryonic selection as a female parent in such crosses eliminates the need for embryo rescue and hybrid progeny can be easily recovered. We have already generated several hundred triploid grapefruit-like hybrids using this approach (J.W. Grosser, unpublished data) using only a few available monoembryonic tetraploid parents.

One approach to overcome the limitation polyembryony imparts is to use the pummelo gene pool in grapefruit breeding. The pummelo is an ideal candidate for development of new grapefruit cultivars because it is one of the ancestors of grapefruit, is a true species, and introduces genetic diversity. Pummelo is monoembryonic and would eliminate the need for embryo rescue when used in interploidal crosses as a female parent. A large range of red-fleshed pummelo selections is available in the Citrus Research and Education Center, Lake Alfred, FL (CREC) germplasm collection that can be used as potential parents. Pummelos can have lower quantities of undesirable compounds such as naringin and furanocoumarins. Thus, when used in crosses, it should be possible to generate desirable seedless hybrids with reduced levels of these compounds.

Colchicine is an alkaloid obtained from Colchicum autumnale, which acts as a mitotic inhibitor (Blakeslee and Avery, 1937) and induces tetraploidy in the target cells by interfering with spindle formation at the metaphase. Colchicine is commonly used to induce tetraploidy in breeding lines in Citrus. Early attempts to generate autotetraploids by treating the axillary buds with colchicine ex vitro were performed by Barrett (1974) in monoembryonic cultivars. However, his technique did not produce any non-chimeric tetraploid plants. Later work showed that autotetraploids can be produced in monoembryonic cultivars from colchicine treatment of axillary buds in vitro (Oiyama and Okudai, 1986) or somatic embryogenic callus (Wu and Mooney, 2002). Recently, we reported on the production of autotetraploid pummelo plants through colchicine treatment of germinating seed (Kainth and Grosser, 2010). However, we are aware of no other reports of tetraploid induction in pummelo at present. This study reports a more efficient method to induce tetraploids by in vitro treatment of cut stem explants from pummelo selections with colchicine followed by shoot induction through indirect organogenesis. The effect of different colchicine concentrations and exposure durations was compared for efficiency of indirect organogenesis and tetraploid induction. 


\section{Materials and Methods}

Plant materials. Pummelo selections 5-199-3, C-HBP, HBJL-5, and HBJL-7 were selected for this study on the basis of their pink or red flesh and other quality characteristics. These selections are all derived from the 'Hirado Buntan' pink pummelo and are located at the experimental farm of the CREC. The seeds were extracted from the mature fruit of each selection, treated with sodium hydroxide for 4 to $5 \mathrm{~min}$ for slime removal, and rinsed three to four times with water. The seeds were dried and refrigerated at $4{ }^{\circ} \mathrm{C}$ until used.

For this experiment, seeds from each selection were peeled and sterilized with $10 \%$ Clorox bleach $(6.25 \%$ sodium hypochlorite) and two drops of Tween-20. Individual seed were put into $15-\mathrm{cm}$ long test tubes containing $15 \mathrm{~mL}$ of solid Murashige and Skoog (MS) medium consisting of MS salts and vitamins (Murashige and Skoog, 1962) supplemented with $30 \mathrm{~g} \cdot \mathrm{L}^{-1}$ sucrose and $7 \mathrm{~g} \cdot \mathrm{L}^{-1}$ agar, $\mathrm{pH}$ 5.8. These were then placed in the dark for $\approx 4$ weeks. The etiolated conditions were provided to facilitate and hasten stem elongation and suppress lateral branching. At $\approx 4$ weeks, when the seedlings attained a height of $\approx 12$ to $15 \mathrm{~cm}$, they were put under continuous light conditions for 4 to $5 \mathrm{~d}$ to allow greening and hardening of the stems. At this time, the seedlings were ready for colchicine treatments.

Colchicine treatments. After hardening of the seedlings, the leaves were removed and each stem was cut into $\approx 1$-cm long explants by making a diagonal cut at both ends. For each selection, colchicine treatments were applied separately. The experiment was designed to examine four colchicine concentrations
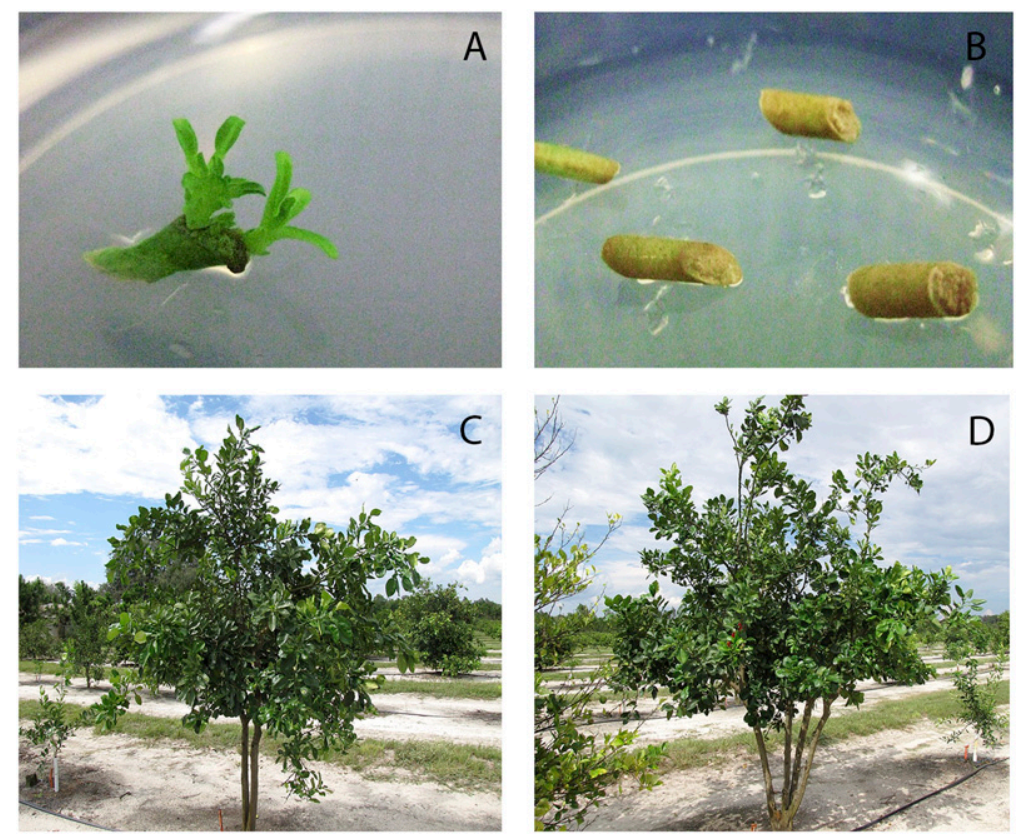

Fig. 1. (A) Indirect organogenesis from callus produced at the cut ends of the stem pieces placed on DBA-3 shoot induction medium. (B) Browning of seedling stem-piece explants caused by higher colchicine concentration and duration. (C) Top-worked tetraploid tree derived from the cybrid Hirado Buntan (CHBP) selection on C-35 citrange rootstock. (D) Top-worked tetraploid tree derived from the 5-1-99-3 selection on C-35 citrange.
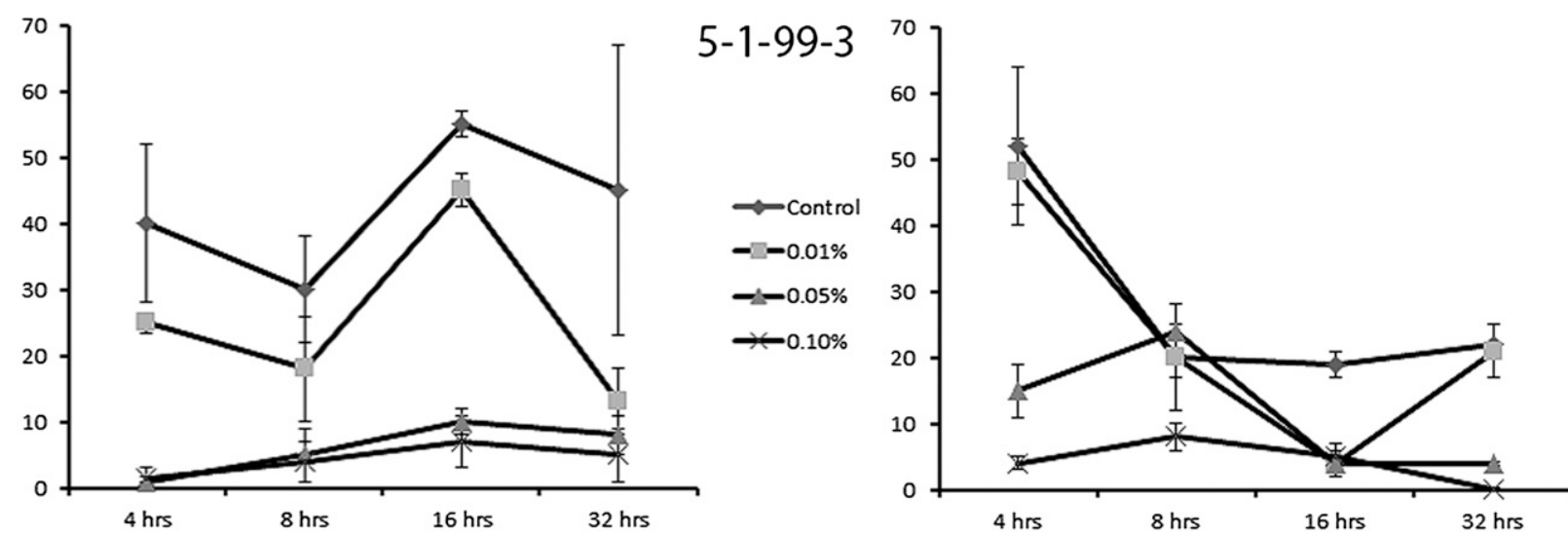

C-HBP
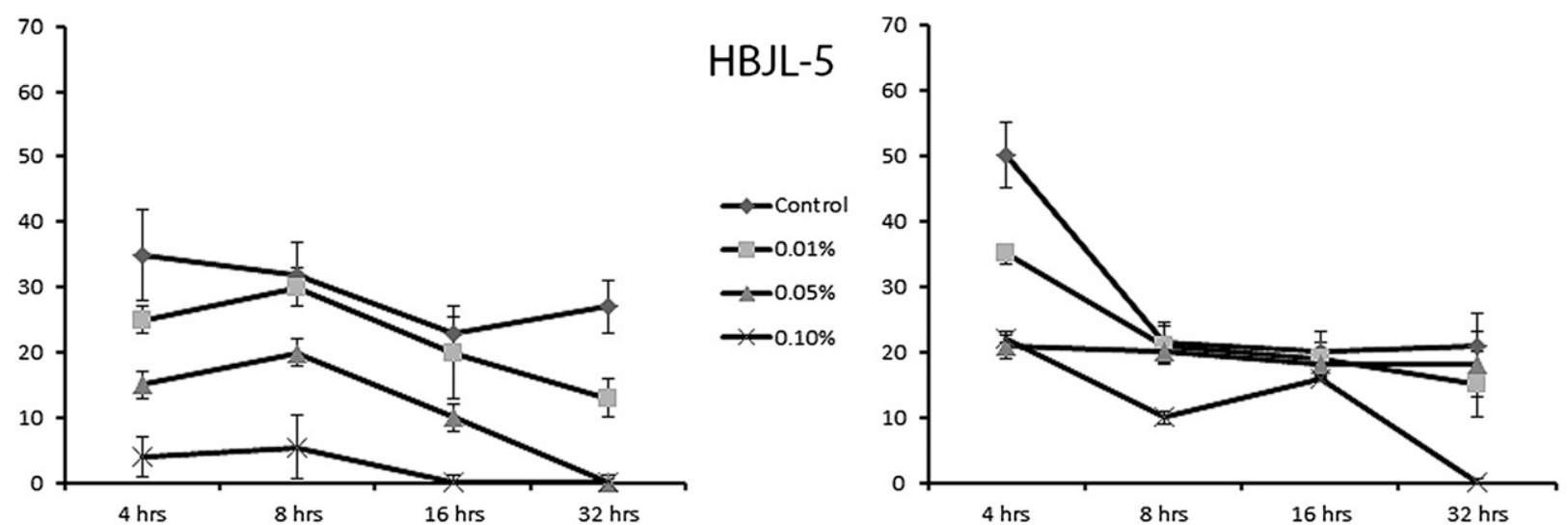

HBJL-7

Fig. 2. Average number of shoots produced through indirect organogenesis from colchicine treatments of pummelo selections 5-1-99-3, cybrid Hirado Buntan (CHBP), HBJL-5, and HBJL-7. Mean separations at each exposure time was obtained by calculating SEM at 5\% level. 
Table 1. Effect of in vitro application of colchicine on the ploidy level of the regenerated shoots.

\begin{tabular}{|c|c|c|c|c|}
\hline $\begin{array}{l}\text { Pummelo } \\
\text { selection }\end{array}$ & $\begin{array}{l}\text { Colchicine } \\
\text { concn }(\%)\end{array}$ & $\begin{array}{c}\text { Exposure } \\
\text { period (days) }\end{array}$ & $\begin{array}{l}\text { No. of tetraploids } \\
\text { produced }\end{array}$ & $\begin{array}{l}\text { No. of mixoploids } \\
\text { produced }\end{array}$ \\
\hline \multirow[t]{12}{*}{$5-1-99-3$} & 0.01 & 4 & 0 & 0 \\
\hline & & 8 & 0 & 0 \\
\hline & & 16 & 3 & 0 \\
\hline & & 32 & 0 & 0 \\
\hline & 0.05 & 4 & 0 & 0 \\
\hline & & 8 & 0 & 0 \\
\hline & & 16 & 0 & 0 \\
\hline & & 32 & 1 & 2 \\
\hline & 0.10 & 4 & 4 & 1 \\
\hline & & 8 & 2 & 0 \\
\hline & & 16 & 0 & 0 \\
\hline & & 32 & 1 & 1 \\
\hline \multirow[t]{12}{*}{ C-HBP } & 0.01 & 4 & 0 & 0 \\
\hline & & 8 & 0 & 0 \\
\hline & & 16 & 0 & 0 \\
\hline & & 32 & 0 & 0 \\
\hline & 0.05 & 4 & 1 & 0 \\
\hline & & 8 & 0 & 1 \\
\hline & & 16 & 2 & 1 \\
\hline & & 32 & 0 & 0 \\
\hline & 0.10 & 4 & 1 & 0 \\
\hline & & 8 & 0 & 0 \\
\hline & & 16 & 0 & 0 \\
\hline & & 32 & 2 & 0 \\
\hline \multirow[t]{12}{*}{ HBJL-5 } & 0.01 & 4 & 0 & 0 \\
\hline & & 8 & 0 & 1 \\
\hline & & 16 & 0 & 0 \\
\hline & & 32 & 0 & 0 \\
\hline & 0.05 & 4 & 0 & 0 \\
\hline & & 8 & 1 & 0 \\
\hline & & 16 & 1 & 1 \\
\hline & & 32 & 0 & 0 \\
\hline & 0.10 & 4 & 0 & 0 \\
\hline & & 8 & 0 & 0 \\
\hline & & 16 & 0 & 0 \\
\hline & & 32 & 0 & 0 \\
\hline
\end{tabular}

C-HBP = cybrid Hirado Buntan.

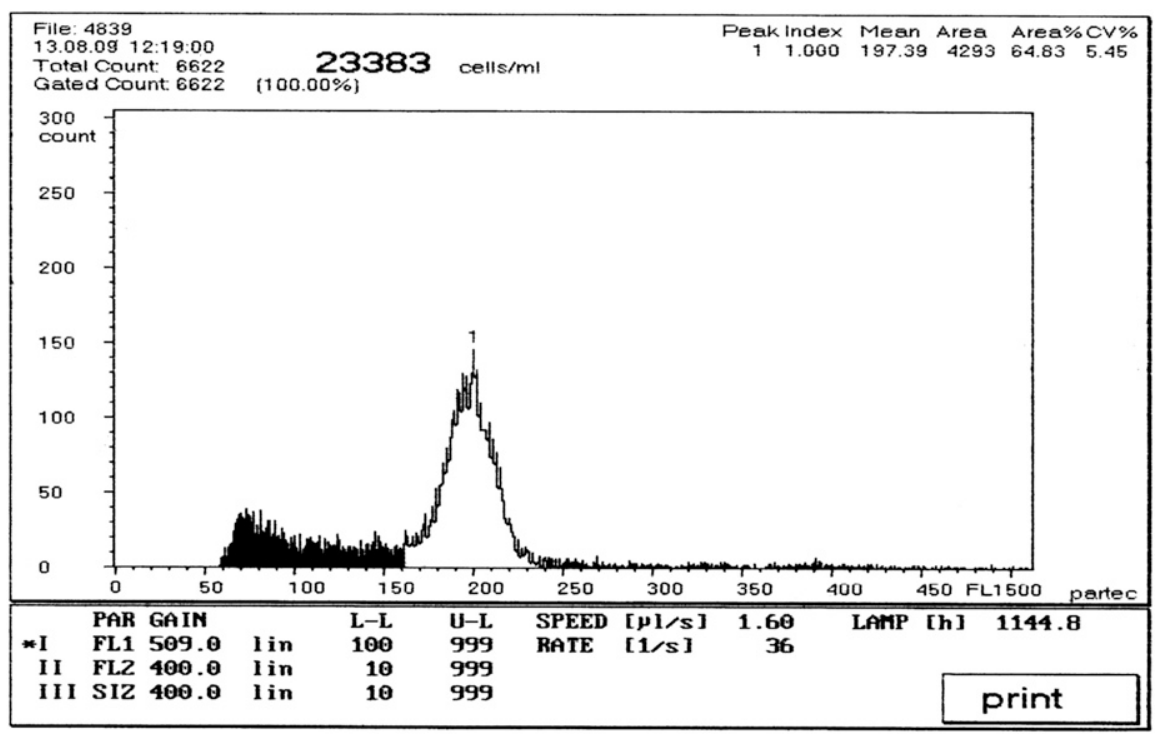

Fig. 3. Flow cytometer peak obtained from a tetraploid shoot of cybrid Hirado Buntan (C-HBP) produced by colchicine treatment in vitro.

A colchicine stock solution was prepared by dissolving the reagent in dimethyl sulfoxide (DMSO) in sterile water to a concentration of $1 \mathrm{~g} \cdot \mathrm{mL}^{-1}$. This solution was filter-sterilized and stored at $-20{ }^{\circ} \mathrm{C}$. The cut explants were incubated in conical Falcon tubes containing
$10 \mathrm{~mL}$ of liquid DBA-3 medium. Colchicine dissolved in DMSO was added to each flask at concentrations of $0.01,0.05$, and $0.1 \%$. DBA-3 shoot induction medium (Deng et al., 1992) contains the cytokinin growth regulator 6-benzylaminopurine in combination with a low level of 2,4-dichlorophenoxyacetic acid, which stimulates cell division and hence increases the frequency of dividing cells available for colchicine exposure. The untreated control treatment consisted of explants immersed in DBA-3 media without colchicine. Each colchicine treatment (different concentration) was performed for four different exposure periods: $4,8,16$, and $32 \mathrm{~h}$. Flasks were placed on the rotary shaker at 30 rpm under conditions of $25 \pm 2{ }^{\circ} \mathrm{C}$ and darkness for the respective treatment duration to facilitate contact and penetration of colchicine into the cells at the cut end.

After the explants were exposed to colchicine, they were placed in $100 \times 20-\mathrm{mm}$ petri dishes on solid DBA-3 medium and were incubated in the dark for 2 weeks to induce callus from the cut ends. The explants were then moved from dark to the continuous light conditions to facilitate shoot induction through indirect organogenesis (Fig. 1A). The explants with emerging shoots from the cut ends were transferred to RMA medium (Grosser and Gmitter, 1990) supplemented with gibberellic acid, which promotes shoot elongation. When shoots were $\approx 1$ to $2 \mathrm{~cm}$ long, individual elongated shoots were moved to RMA rooting medium in Magenta boxes for further hardening and growth. Emergent shoots were analyzed for their ploidy level using a flow cytometer at a stage when the shoots had approximately three expanded leaves. The tetraploid and mixoploid shoots confirmed by flow cytometry were propagated by micrografting onto vigorous trifoliate rootstocks. The micrografted mixoploids and tetraploids were grown under shade for 10 to $14 \mathrm{~d}$ after which they were moved to the greenhouse with 21 to $17{ }^{\circ} \mathrm{C}$ day-night temperatures for acclimatization.

Ploidy analysis. Ploidy level analysis was performed using a tabletop Ploidy Analyser flow cytometer (Partec GmbH, Germany) according to Dutt et al. (2010) as briefly described. Leaf pieces $\left(0.2\right.$ to $\left.0.3 \mathrm{~cm}^{2}\right)$ of fully expanded leaf tissue were excised and placed in a $50-\mathrm{mm}$ plastic petri dish. The samples were chopped with a sharp razor blade after adding few drops of Nuclei Extraction Buffer. After chopping, six to seven additional drops of Nuclei Extraction Buffer were added and the samples were filtered through a $50-\mu \mathrm{m}$ filter into a $3.5-\mathrm{mL}(55 \mathrm{~mm} \times 12$ $\mathrm{mm})$ Sarstedt tube. The staining buffer $\left(4^{\prime}, 6-\right.$ diaminidino-2-phenylindole) was added drop by drop through the filter to infiltrate the remaining cells until half of the tube was filled. Each sample was incubated for 10 to $15 \mathrm{~s}$ at room temperature before analyses.

\section{Results and Discussion}

In this study, a method to induce tetraploidy in non-apomictic seedlings by treating cut stem sections with colchicine at various concentrations and exposure periods is described. Colchicine had a negative effect on shoot regeneration at all concentrations and all exposure periods. This is attributed to the toxic nature of colchicine. The colchicine 


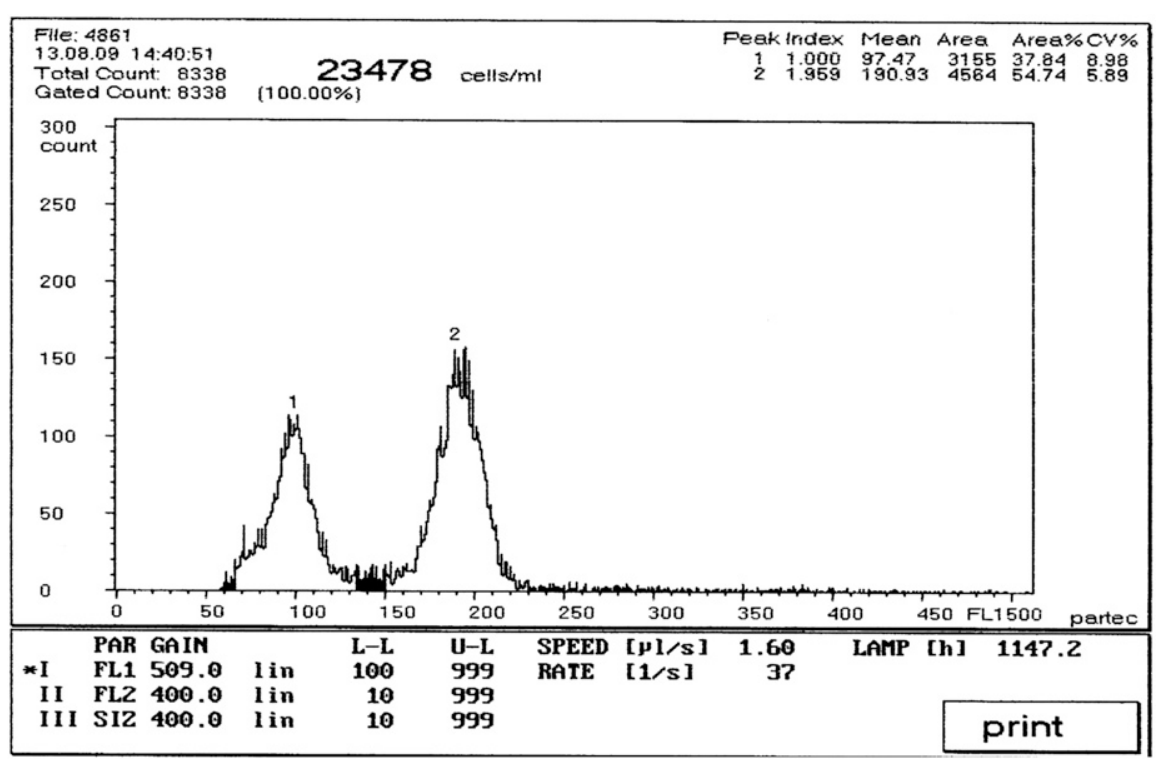

Fig. 4. Flow cytometer peaks (diploid and tetraploid) obtained from a mixoploid shoot of cybrid Hirado Buntan (C-HBP) produced by colchicine treatment in vitro.

concentration and the exposure time of the explants were the key factors affecting indirect organogenesis as well as generation of tetraploids. These two factors have been recognized previously as the two main parameters affecting tetraploid production by Sanford (1983). The shoot induction was through indirect organogenesis, i.e., from the callus produced from the cut ends of the treated explants. Colchicine treatments also affected indirect organogenesis adversely. The explants from treatments with higher concentrations of colchicine or longer exposure periods did not produce as much callus at the cut ends as compared with the untreated control and the lower concentration and duration treatments. Callus production at the cut ends is a healing response of the tissue to the injured cells at the cut. Colchicine seemed to impair the ability of the stem tissue to heal by inhibiting callus production at the injury sites. The explants from the treatments with higher concentrations and longer exposure periods showed browning as well as necrosis (Fig. 1B). Browning prevented the explants from producing callus and subsequent shoots. Such explants turned from light brown to dark brown and eventually died.

The rate of shoot induction through indirect organogenesis decreased progressively with the increase in the concentration and duration of the colchicine treatments. Treatments with higher concentrations of colchicine or longer exposure periods had significantly fewer shoots as compared with those from control treatments or less severe treatments. The decrease in shoot induction is probably the result of decreased callus production in the severe treatments. Reduced callus production provides less active surface area for shoot formation. Also, colchicine toxicity caused mortality of the cells, which resulted in lower callus formation and subsequent shoot induction. In all the selections, the $0.01 \%$ treatment with 4 -h exposure duration produced the maximum average number of shoots ranging from $\approx 25$ to 50 . The highest number of shoots for this treatment was produced in the C-HBP selection. Lowest numbers of shoots were produced from treatments with the highest colchicine concentration of $0.1 \%$ and maximum exposure of $32 \mathrm{~h}$. This treatment produced one to five shoots emerging from the explants in all the selections except in selection HBJL-5 where it totally suppressed shoot induction. (Fig. 2). An analysis of variance of these data revealed that $16 \mathrm{~h}$ of exposure produced significantly more shoots for all concentrations (data not presented).

Indirect organogenesis from the cut ends of the explants allowed for more efficient induction of polyploidy in the target cells. Selections 5-1-99-3, C-HBP, and HBJL-5 produced 11 , six, and two tetraploids and four, two, and two mixoploids, respectively. HBJL-7 failed to produce any polyploid plants (Table 1). It was observed that the higher levels of colchicine $(0.05 \%$ and $0.1 \%)$ produced the most tetraploid shoots. We did not observe a trend in the exposure period to the rate of tetraploid production and it varied according to the pummelo selection. In total, we obtained 19 tetraploid shoots from the C-HBP, 5-1-99-3, and HBJL-5 pummelo selections. Of the 19 stable tetraploid shoots produced, 10 were obtained from the treatment with a concentration of $0.1 \%$ and six from the treatment with a $0.05 \%$ colchicine concentration. Although only a few tetraploids were produced from treatments with the lowest colchicine concentration of $0.01 \%$, these treatments were more effective at longer durations. A $0.1 \%$ concentration of colchicine was observed to be most efficient for tetraploid induction. This colchicine concentration was also recognized as optimal for treatment of citrus shoot tips for tetraploid induction by Oiyama and Okudai (1986). The differential response of the selections in the number of regenerated plants can be explained by the variation in the susceptibility of the genotypes to colchicine. Such genotypic effects leading to variable results have been reported previously (Aleza et al., 2009; Ganga and Chezhiyan, 2002; Stanys et al., 2006). Figure 3 displays the histogram obtained from the cell cytometer for a tetraploid sample showing a single tetraploid peak, whereas Figure 4 displays a histogram for a mixoploid sample showing two peaks depicting the presence of diploid and tetraploid ploidy levels in the same sample. The tetraploids were propagated by micrografting to vigorous trifoliate rootstock seedlings. Mixoploids were discarded because they are unstable, often sterile, and of little use for the breeding process. In an earlier study, we described a method to induce tetraploidy in pummelo seedlings by treating pre-germinated seeds with colchicine at various concentrations and exposure periods (Kainth and Grosser, 2010). This protocol described here, however, is more efficient and resulted in a lower number of mixoploids. This is the result of the indirect organogenesis system used here as compared with direct organogenesis reported earlier.

\section{Conclusion}

The results from this study demonstrated successful production of non-chimeric tetraploid plants from monoembryonic pummelo selections selected for their red flesh and superior quality. Stable tetraploids were successfully produced in three of four selections and were confirmed by flow cytometry. These tetraploids were top-worked to young field trees of C-35 citrange rootstock to expedite flowering and fruiting (Fig. 1C-D). The tetraploids obtained could be of significant value as parents in our grapefruit/pummelo triploid breeding program, especially if any exhibit canker tolerance and good fruit quality.

\section{Literature Cited}

Aleza, P., J. Juarez, P. Ollitrault, and L. Navarro. 2009. Production of tetraploid plants of non apomictic citrus genotypes. Plant Cell Rpt. 28:1837-1846.

Barrett, H.C. 1974. Colchicine-induced polyploidy in citrus. Bot. Gaz. 135:29-41.

Barrett, H.C. and A.M. Rhodes. 1976. A numerical taxonomic study of affinity relationships in cultivated Citrus and its close relatives. Syst. Bot. 1:105-136.

Blakeslee, A.F. and A.G. Avery. 1937. Methods of inducing doubling of chromosomes in plants by treatment with colchicine. J. Hered. 28:393412.

Cameron, J.W. and R.H. Burnett. 1978. Use of sexual tetraploid seed parents for production of triploid Citrus hybrids. HortScience 13:167169.

Chen, R.Z., G.G. Li, and L.Y. Zhang. 1991. Callus induction and triploid plant regeneration from endosperm of 'Hongjiang' sweet orange. Acta Bot. Sin. 33:848-854

Deng, X.X., J.W. Grosser, and F.G. Gmitter, Jr. 1992. Intergeneric somatic hybrid plants from protoplast fusion of Fortunella crassifolia 
cultivar Meiwa with Citrus sinensis cultivar Valencia. Sci. Hort. 49:55-62.

Deng, X.X., G.B. Liu, and W.C. Zhang. 1985. Studies on the chromosome variation in the callus of citrus. China Citrus 3:4-6.

Dutt, M., M. Vasconcellos, K.J. Song, F.G. Gmitter, Jr., and J.W. Grosser. 2010. In vitro production of autotetraploid Ponkan mandarin (Citrus reticulata Blanco) using cell suspension cultures. Euphytica 173:235-242.

Esen, A. and R.K. Soost. 1971. Unexpected triploids in Citrus: Their origin, identification, and possible use. J. Hered. 62:329-333.

Esen, A. and R.K. Soost. 1972. Tetraploid progenies from $2 \mathrm{x} \times 4 \mathrm{x}$ crosses of Citrus and their origin. J. Amer. Soc. Hort. Sci. 97:410-414.

Ganga, M. and N. Chezhiyan. 2002. Influence of the antimitotic agents colchicine and oryzalin on in vitro regeneration and chromosome doubling of diploid bananas (Musa spp.). J. Hort. Sci. Biotechnol. 77:572-575.

Geraci, G. 1978. Percentage of triploid offspring of cross-pollinated diploid polyembryonic citrus. Proc. Intl. Soc. Citricult. 1:57-58.

Geraci, G., F. De Pasquale, and N. Tusa. 1977. Percentages of spontaneous triploids in progenies of diploid lemons and mandarins. Proc. Intl. Soc. Citricult. 2:596-597.

Geraci, G., A. Esen, and R.K. Soost. 1975. Triploid progenies from $2 \mathrm{x} \times 2 \mathrm{x}$ crosses of Citrus cultivars. J. Hered. 66:177-178.
Gmitter, F.G., Jr. 1995. Origin, evolution and breeding of the grapefruit. Plant Breed. Rev. 13:345-363.

Grosser, J.W. and F.G. Gmitter, Jr. 1990. Protoplast fusion and citrus improvement. Plant Breed. Rev. 8:339-374.

Grosser, J.W. and F.G. Gmitter, Jr. 2011. Protoplast fusion in the production of tetraploids and triploids: Applications in scion and rootstock breeding. Plant Cell Tis. Org. Cult. 104:343-357.

Kainth, D. and J.W. Grosser. 2010. Induction of autotetraploids in pummelo (Citrus grandis $\mathrm{L}$. Osbeck) through colchicine treatment of meristematically active seeds in vitro. Proc. Fla. State Hort. Soc. 123:44-48.

Kobayashi, S., T. Ohgawara, W. Saito, Y. Nakamura, and M. Omura. 1997. Production of triploid somatic hybrids in citrus. J. Jpn. Soc. Hort. Sci. 66:453-458.

Koltunow, A.M., P. Brennan, J.E. Bond, and S.J. Barker. 1998. Evaluation of genes to reduce seed size in Arabidopsis and tobacco and their application to Citrus. Mol. Breed. 4:235-251.

Murashige, T. and F. Skoog. 1962. A revised medium for rapid growth and bio-assays with tobacco tissue cultures. Physiol. Plant. 15:473-497.

Oiyama, I. and N. Okudai. 1986. Production of colchicine-induced autotetraploid plants through micrografting in monoembryonic Citrus cultivars. Jap. J. Breed. 36:371-376.
Sanford, J.C. 1983. Ploidy manipulations, p. 100-123. In: Moore, J.N. and J. Janick (eds.). Methods in fruit breeding. Purdue Univ. Press, West Lafayette, IN.

Scora, R.W. 1975. On the history and origin of citrus. Bull. Torrey Bot. Club 102:369-375.

Scora, R.W., J. Kumamoto, R.K. Soost, and E.M. Nauer. 1982. Contribution to the origin of the grapefruit, Citrus paradisi (Rutaceae). Syst. Bot. 7:170-177.

Soost, R.K. and J.W. Cameron. 1975. Citrus. Advances in fruit breeding. Subtrop. Fruits. p. 507-540.

Stanys, V., A. Weckman, G. Staniene, and P. Duchovskis. 2006. In vitro induction of polyploidy in Japanese quince (Chaenomeles japonica). Plant Cell Tis. Org. Cult. 84:263-268.

Wakana, A., M. Iwamasa, and S. Uemoto. 1982. Seed development in relation to ploidy of zygotic embryo and endosperm in polyembryonic citrus. Proc. Intl. Soc. Citicult. 1:3539.

Wang, T.Y. and C.J. Chang. 1978. Triploid citrus plantlet from endosperm culture. Sci. Sin. 21:823-827.

$\mathrm{Wu}, \mathrm{J}$. and P. Mooney. 2002. Autotetraploid tangor plant regeneration from in vitro Citrus somatic embryogenic callus treated with colchicine. Special Issue on the New Zealand Regional IAPTC \& B Conference, Mount Ruapehu, New Zealand 70:99-104. 